DOI:10.12737/article_5ab0dfbe6ece23.91630316

УДК 630*383-025,13(043)

ФОРМИРОВАНИЕ МОДЕЛИ ПРОЕКТИРОВАНИЯ СИСТЕМЫ «ДОРОЖНЫЕ УСЛОВИЯ - ТРАНСПОРТНЫЕ ПОТОКИ» И ПУТИ ЕЕ РЕАЛИЗАЦИИ

\author{
кандидат технических наук, доцент В. Г. Козлов ${ }^{1}$ \\ доктор технических наук, профессор А. В. Скрыпников ${ }^{2}$ \\ экстерн Е. Ю. Микова ${ }^{2}$ \\ экстерн Р. В. Могутнов ${ }^{2}$ \\ экстерн Е. В.Чирков 2 \\ 1 - ФГБОУ ВО «Воронежский государственный аграрный университет имени императора Петра I», \\ г. Воронеж, Российская Федерация \\ 2 - ФГБОУ ВО «Воронежский государственный университет инженерных технологий», \\ г. Воронеж, Российская Федерация
}

\begin{abstract}
Предложена модель управления системой «дорожные условия -транспортные потоки»позволяющая обеспечивать функционирование комплекса «водитель - автомобиль - дорога - среда» в любых условиях погоды и климата. Анализ предложенной модели и целевого функционала показывает, что оптимизация функционирования системы «дорожные условия -транспортные потоки»может идти по многим направлениям, главными из которых являются: оптимальное развитие и размещение сети лесовозных автомобильных дорог, совершенствование их технического уровня и уровня содержания, инженерное оборудование дорог, эффективное управление дорожным движением, исключая рациональное распределение лесовозных транспортных потоков по сети лесовозных автомобильных дорог и управление режимами на отдельных лесовозных автомобильных дорогах и участках лесовозных автомобильных дорог.Разработанная модель позволяет осуществить теоретический анализ и экспериментальную проверку взаимодействия систем комплекса «водитель - автомобиль - дорога - среда» с целью выявления резервов повышения эффективности его функционирования и целенаправленного управления этим процессом. Транспортно-эксплуатационные характеристики лесовозных автомобильных дорог являются главными факторами, которые формируют условия движения лесовозов на дороге, режим, удобство и безопасность движения и определяют эффективность работы автомобильного транспорта, причем связи многих характеристик дорог с режимом движения носят детерминированный, коррекционный характер. Эти закономерности являются базой для разработки технических требований к лесовозным автомобильным дорогам.Предложенная модель комплексного создания и управления функционированием лесовозной автомобильной дороги может внедриться уже в настоящее время. Однако при проектировании дорог целесообразно предусматривать комплекс управляющих инженерных и организационных мероприятий, обеспечивающих расчетные условия движения в процессе всего периода эксплуатации дороги, независимо от периода года и погодно-климатических условий.Оценка эффективности принятых решений должна производиться с обязательным учетом обеспечения требуемых показателей работы лесовозной автомобильной дороги в неблагоприятные периоды года и в сложных погодных условиях.
\end{abstract}

Ключевые слова: лесовозная дорога, модель, функционирование, дорожные условия, транспортные потоки, автомобиль. 


\title{
FORMATION OF THE MODEL OF DESIGNING THE SYSTEM "ROAD CONDITIONS - TRANSPORT FLOWS" AND WAYS OF ITS IMPLEMENTATION
}

\author{
$\mathrm{PhD}$ (Engineering), Associate Professor V.G. Kozlov ${ }^{1}$ \\ DSc (Engineering), Professor A.V. Skrypnikov ${ }^{2}$ \\ External student E.Yu. Mikova ${ }^{2}$ \\ External student R.V. Mogutnov ${ }^{2}$ \\ External student E.V. Chirkov 2 \\ 1 - FSBEI HE "Voronezh State Agrarian University named after Emperor Peter I", \\ Voronezh, Russian Federation \\ 2 - FSBEI HE "Voronezh State University of Engineering Technologies", Voronezh, Russian Federation
}

\begin{abstract}
A model for managing the system "road conditions - traffic flows" is proposed that allows to ensure the functioning of the complex "driver - car - road - environment" in any weather and climate conditions. The analysis of the proposed model and target functional shows that optimization of the "road conditions - traffic flow" system can proceed in many directions, the main of which are: optimal development and placement of the network of forest roads, improving their technical level and level of maintenance, road engineering equipment, efficient traffic management, excluding the rational distribution of forest traffic on the network of forest roads and the management modes on individual logging roads and sections of forest roads. The developed model makes it possible to carry out a theoretical analysis and experimental verification of the interaction between «driver-car-road-environment" system in order to identify the reserves of increasing efficiency of its operation and purposeful management of this process. Transport-operational characteristics of logging roads are the main factors that shape the conditions of timber transport on the road, mode, convenience and safety of traffic and determine the efficiency of road transport, and connections of many characteristics of roads with the traffic modes have deterministic, corrective character. These patterns are the basis for the development of technical requirements for logging roads. The proposed model for integrated creation and management of the functioning of a forest road may already be implemented. However, the design of roads is expedient to provide complex control engineering and organizational measures to ensure the estimated traffic conditions during the whole period of operation of the road, regardless of the time of year and weather and climate conditions. Evaluation of the effectiveness of taken decisions should be made with mandatory consideration for ensuring the required performance of logging road in adverse periods of the year and in difficult weather conditions.
\end{abstract}

Keywords: logging road, model, operation, road conditions, traffic flows, vehicle.

В настоящее время исследованию функционирования комплекса «водитель - автомобиль дорога - среда» ВАДС и отдельных его систем посвящено большое количество работ $[4,5]$ в которых дан большой анализ влияния транспортных потоков, дорожных условий и организации движения на эффективность работы лесовозного автомобильного транспорта, режим и безопасность движения. В большинстве указанных работ рассматривается комплекс «водитель - автомобиль - дорога» (ВАД), в некоторых из них обращается внимание на необходимость учета влияния внешних факторов [6].

Считая известными затраты на приведение параметров дороги в соответствие с требованиями движения - Н (Д - дорога) и затраты на улучшение содержания дороги для пропуска транспортного потока с расчетной интенсивностью $\mathrm{G}$ (Д), а также располагая экономическими показателями комплекса ВАДС-функциями F, H, G - можно сформулировать технико-экономическую задачу об оптимальных условиях функционирования комплекса ВАДС в общем виде. Будем считать, что задана совместная функция распределения параметров транспортного потока и окружающей среды в годе $\gamma \mathrm{t}\left(\mathrm{T \Pi}_{1} \mathrm{C}\right) \mathrm{dT} \Pi_{1 i}, \ldots, \mathrm{dT}_{n} \mathrm{dC}_{1}, \ldots, \mathrm{C}_{m}$, которая 
выражает долю часов в году, когда параметры транспортного потока находятся в пределах. $\mathrm{TП}_{i}$, ТП $_{i}+\mathrm{dTПпри} \mathrm{состоянии} \mathrm{среды} \mathrm{в} \mathrm{пределах} \mathrm{C}_{i}=$ лах $\mathrm{C}_{i}=\mathrm{C}_{i}+\mathrm{dC}_{i}$. Зависимость этой функции от года $\mathrm{t}$ связана с изменением погодных условий и параметров транспортного потока. Функция распределения параметров автомобилей $\gamma_{1}(\mathrm{~A})$ зависит от $\mathrm{t}$, так как происходит постоянное совершенствование и обновление парка автомобилей, и $\gamma(\mathrm{B})-$ функция распределения параметров водителей. Для выбора оптимальной программы капиталовложений, необходимо учесть одновременные затраты $\mathrm{H}$ $(\Delta \mathrm{D})$ на совершенствование качеств дороги $\Delta \mathrm{D}$ и затраты $\left(\overline{D_{t}}\right)$ на содержание дороги. Соответствующее приращение штрафной функции (отрицательное) будет:

$$
\Delta F=\frac{\partial F}{\partial D} \Delta D+\frac{\partial F}{\partial D} \Delta \overline{D_{t}}
$$

При данном состоянии среды С, целевая функция будет выглядеть следующим образом:

$$
\begin{aligned}
S= & \sum_{t}\left\{\left(\int _ { B } \int _ { A } \int _ { \mathrm { T } \Pi } \int _ { C } \left(\frac{\partial F}{\partial D} \Delta D\right.\right.\right. \\
& \left.+\frac{\partial F}{\overline{\partial D}} \Delta \overline{D_{t}}\right) \varphi_{t}(A, B, \mathrm{~T} \Pi, C) \partial D \cdot \partial C \\
& \left.\cdot \partial B \cdot \partial \mathrm{T} \Pi)+G\left(\Delta \overline{D_{t}}\right)\right\} e^{-\varepsilon t} \\
& +H(\partial D) \rightarrow \min
\end{aligned}
$$

где $\varepsilon=\ln \left(I+E_{n}\right)$;

$E_{n}-$ коэффициент приведения.

При этом необходимо учесть условия ограниченности ресурсов $G\left(\Delta \overline{D_{t}}\right) \geq g_{t}$

$$
\mathrm{H}(\Delta \mathrm{D}) \leq \mathrm{h}
$$

Решение экстремальной задачи (2) дает возможность оптимизировать параметры всех систем комплекса с учетом возмущающих воздействий окружающих условий по принятому критерию достижения конечной цели. Так же как программы по развитию и совершенствованию других систем, программа деятельности по строительству, реконструкции $\Delta \mathrm{D}$, ремонту, содержании дорог и организации движения $\Delta D_{p}, \Delta \mathrm{DC}$ будет оптимальной, если она соответствует $S_{\min }$.

Решению задачи (2) препятствуют трудности теоретического и практического характера, главной из которых является отсутствие достаточной исходной информации о составляющих комплекса и их совместном распределении. Однако имеются все предпосылки для решения задачи оценки эффективности мероприятий по повышению технического уровня содержания конкретной дороги или ее участка, а также для сравнения вариантов этих мероприятий. Эта задача может и должна решаться на стадии проектирования дорог в следующей последовательности:

1. Определяются параметры, характеризующие транспортный поток и окружающую среду, возможные и допустимые пределы их колебаний, степень детализации их членения. Пространство ТП, С может быть описано координатами Z1, Z2, C1, C2, C3, С4 и т.д., где Z1, Z2 - интенсивность и состав движения; C1, C2, C3, C4 - признаки действия метеорологических явлений.

2. Оцениваются численные значения $\varphi$ (ТП, С), т.е. описываются изменения интенсивности движения по сезонам года и по состоянию погоды внутри сезонов.

3. Намечаются конкурирующие варианты мероприятий по снижению движения транспортного потока и окружающей среды на режим движения для длинного участка дороги, и определяется стоимость $\Delta \mathrm{D}$ и $\Delta \overline{D_{i}}$ для каждого варианта, включая и нулевой. Для существующей дороги за нулевой принимается вариант без дополнительных капиталовложений, а для проектируемой - вариант с минимальными капитальными затратами.

4. Вычисляется приращение штрафной функции $\Delta \mathrm{F}$ в каждой точке пространства ТП, С. Для этого необходимо разработать методику определения потерь от снижения скоростей движения и от увеличения ДТП при ухудшении условий движения по сравнению с эталонами.

5. Выбрав сроки сравнения и коэффициенты приведения, определяют значения целевой функции.

Таков путь определения техникоэкономической эффективности мероприятий по улучшению взаимодействия комплекса ВАДС, его подсистем, путь эффективного управления транспортно-эксплуатационными характеристиками лесовозных автомобильных дорог с учетом различия 


\section{Лесоинженерное дело}

климата и погоды по регионам лесозаготовок страны при проектировании.

Разработка указанного направления представляется весьма перспективной, поскольку решение проблемы эффективного управления дорожной сети, техническим совершенством и состоянием дорог с учетом климата и погоды позволяет с одной стороны без изменения общей суммы капитальных вложений и текущих затрат в дорожную сеть, получить наибольший экономический эффект за счет обоснованного планирования и распределения материально-технических ресурсов на эти цели, а с другой стороны более обосновано определять необходимые ресурсы на развитие и совершенствование дорожной сети регионов, с учетом возможных потерь от несовершенства автомобильно-дорожной системы в неблагоприятные периоды года и в сложных погодных условиях.

Предложенный функционал 2 является целевым для модели управления транспортноэксплуатационными характеристиками лесовозных автомобильных дорог и режимами движения лесовозных транспортных потоков при возмущающихся воздействиях метеорологических факторов в различных дорожных и климатических условиях.

В соответствии с принципами системного анализа и теории операций $[3,7]$, указанная модель должна объективно отражать взаимодействия систем комплекса ВАДС, сущность исследуемой операции и соответствовать цели исследования.

Прежде чем перейти к конструированию модели, необходимо рассмотреть вопрос о терминологии. Основные определения элементов комплекса ВАДС даны в работах Бабкова В.Ф., Иванова В.Н., Калужского Я.А., Клинковштейна Г.И., Сиденко В.М., Сильянова В.В., Хомяка Я.В., Курьнова В.К., Скрыпникова А.В., Кондрашовой Е.В. и др. $[1,2,4,10]$. Однако большинство из указанных предложений не охватывает всех параметров комплекса ВАДС.

Исходя из целей исследования, нами предложена развернутая модель функционирования комплекса ВАДС и приняты ниже следующие понятия и основные предложения (рис. 1).
Дорожные условия (ДУ) - совокупность геометрических параметров и транспортноэксплуатационных качеств дороги, имеющих непосредственное отношение к движению, подразделяется на постоянные переменные (временные и кратковременные) параметры и факторы.

К постоянным относятся параметры и характеристики дорог, которые не меняются в процессе эксплуатации или изменяются очень редко (во время реконструкции или капитального ремонта). Это параметры продольного профиля, радиусы в плане, длины прямых и кривых и др.

К переменным (временным или сезонным) факторам отнесены параметры и характеристики дорог, изменяющиеся в течении года в результате сезонных колебаний метеорологических условий и качества содержания дороги: состояние покрытия и обочин, ровность и сцепные качества покрытия, фактическая ширина проезжей части и обочин, наличие и состояние съездов и пересечений, состояние инженерного оборудования дорог, видимость в плане и др.

К переменным кратковременным относятся факторы, влияющие на режим и безопасность движения в течение короткого времени - от нескольких часов до одного месяца: осадки, туман, гололед, ветер, метеорологическая видимость и др.

Транспортный поток (ТП) - совокупность отдельных автомобилей $A$ на дороге, каждым из которых управляет водитель В. Транспортный поток характеризуется интенсивностью и составом. Транспортный поток также является управляемой системой комплекса, так как характеристики транспортного потока могут регулироваться (ограничением въезда определенных автомобилей, перенос движения на параллельные маршруты и полосы движения во времени и т. д.).

Состояние окружающей среды (C) - совокупность метеорологических поступков или условий погоды в данный момент времени. Метеорологические условия характеризуются значениями метеорологических элементов (явлений, факторов), за которыми ведутся наблюдения [4]. 


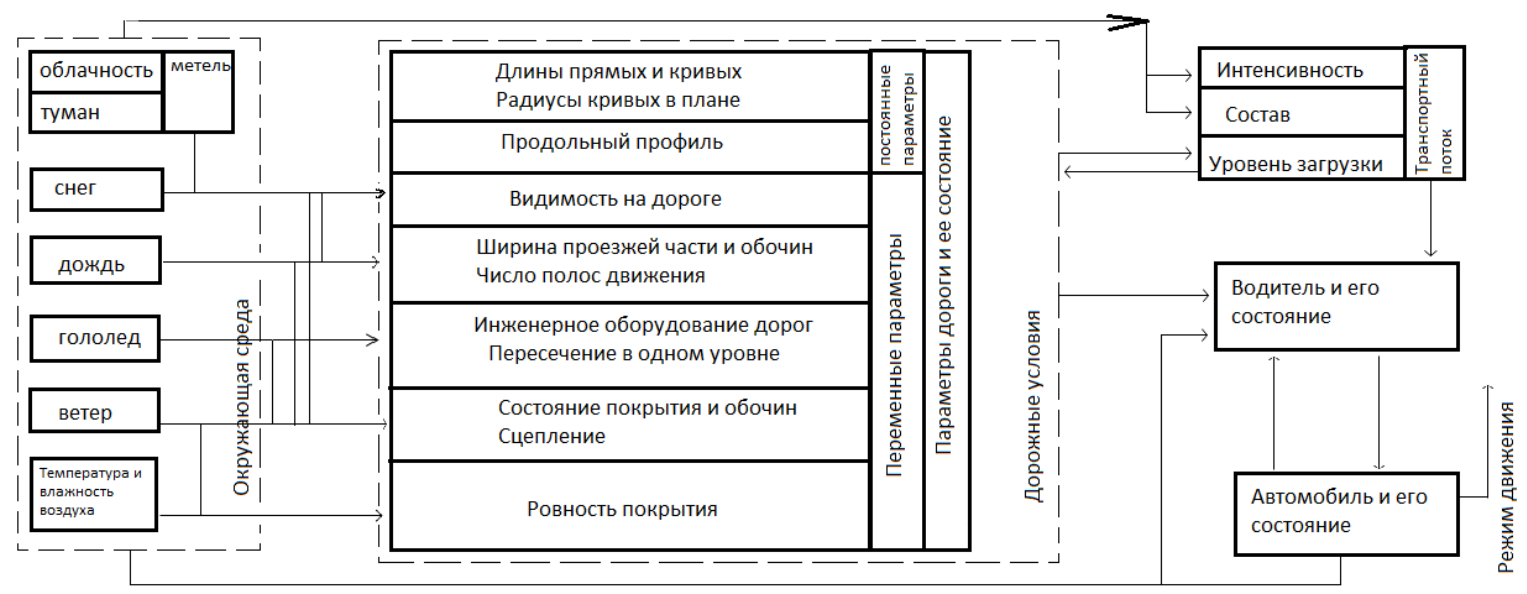

Рис. 1. Схема взаимодействия систем и факторов комплекса «водитель-автомобиль-дорога-среда»

Изменения метеорологических условий носят случайный, неуправляемый характер, хотя они связаны с общими природно-климатическими характеристиками регионов, которые изменяются по определенным закономерностям во времени и в пространстве. Неопределенность метеорологических условий придает характер неопределенности функционированию всего комплекса ВАДС.

Наибольшее влияние оказывают метеорологические условия на переменные параметры, характеристики дорог и режимы движения. Можно выделить метеорологические элементы и их сочетания с немедленным воздействием (осадки, гололед, туман, ветер) и с накопленным воздействием (образование пучин, снежно-ледяных отложений).

Режим движения характеризуется скоростью движения одиночных автомобилей и всего потока, интервалами между автомобилями в потоке, плотностью потока, количеством обгонов, перестроений и их траекториями, режимом разгонов и торможений. Режим движения является важнейшей характеристикой функционирования всего комплекса и интегрально отражает его эффективность и качество.

Анализ взаимодействия систем показывает, что основными управляемыми элементами комплекса являются дорожные условия и транспортные потоки, а также их взаимодействие, поскольку параметры подсистемы «водитель-автомобиль» изменяются менее динамично и на определенном этапе могут быть приняты среднестатистическими.
Стержнем дальнейших исследований принято положение о том, что комплекс ВАДС и система «дорожные условия - транспортные потоки» (ДУТП) является двухуровневой децентрализованной системой обслуживания со случайными стохастическими входами, включающими спрос на использование дорогой со стороны транспортного потока и возмущающие воздействие окружающей среды.

Основываясь на принятой концепции, нами предложена модель двухуровневого управления функционированием системы ДУ-ТП применительно к одной дороге или сети дорог $[4,5,9]$.

Первый уровень - стратегическое или программное управление, которое осуществляется путем выбора и назначения технических параметров и транспортно-эксплуатационных характеристик лесовозной автомобильной дороги на стадии проектирования. Сюда же относятся капитальные мероприятия по организации движения, а также выбор и обоснование форм и методов содержания дороги, выбор и обоснование необходимой мощности и ресурсов дорожно-эксплуатационной службы.

Второй уровень - оперативное или текущее управление, которое осуществляется на уровне процесса обслуживания дорожными условиями транспортных потоков. Оперативное управление функционированием системы ДУ-ТП осуществляется мероприятиями по содержанию дорог и организации движения. При этом существенное значение имеют основные технические характеристики лесовозной автомобильной дороги, фазовые переменные подсистемы «дорожные условия», как ха- 


\section{Лесоинженерное дело}

рактеристики аппарата обслуживания, принятые на первом уровне управления.

Принципиальная схема управления в системе ДУ-ТП представлена на рисунке 2, где показано, что при заданных постоянных параметрах дороги, организации движения и уровне содержания дороги под влиянием окружающей среды и транспортного потока формируется фактическое состояние дороги, режимы движения и уровень безопасности, т.е. процесс обслуживания.

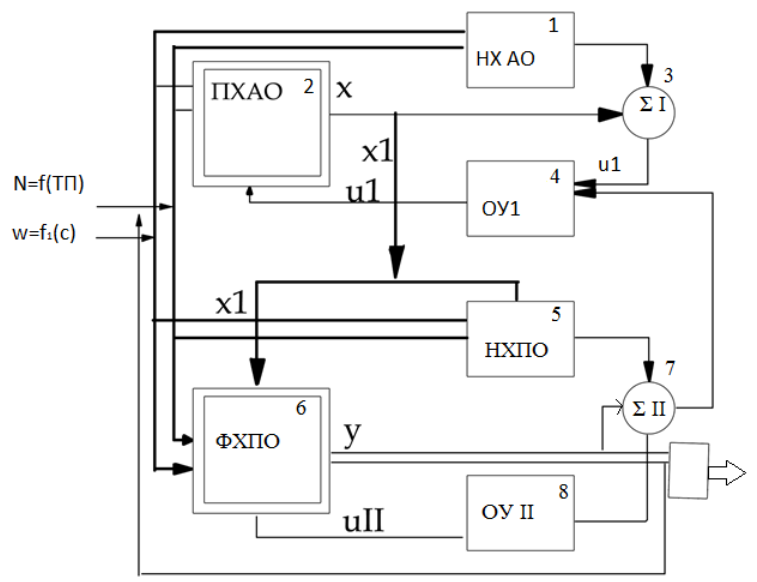

Рис. 2. Блок-схема управления в системе ДУ - ТП: $\mathrm{N}$ - спрос на пользование автомобильной дорогой со стороны транспортного потока; $\mathrm{w}$ - распределение характеристик метеорологических условий; UI и UII - соответственно векторы управления I и II уровня; блоки 1 и 2 - соответственно нормативные и проектные характеристики аппарата управления; блоки 3 и 7 сумматоры 1 и 2 уровня управления; блоки 4 и 8 - блоки определения оптимального управления 1 и 2 уровня; блоки 5 и $6-$ соответственно нормативные и фактические характеристики процесса обслуживания.

В тоже время для заданных условий: той же интенсивности ТП, метеорологические условия С и заданных постоянных параметров дороги существует нормативное (требуемое) состояние переменных параметров дорожных условий и организации движения, при которых характеристики процесса обслуживания наиболее оптимальны.

Сравнивая фактические характеристики процесса обслуживания и его выходные характеристики с требуемыми или нормативными для данных условий, в сумматоре определяется рассогласование $\mathrm{Y}^{-} \mathrm{Y}^{\wedge}$, в зависимости от величины этого рассогласования назначается управление второго, либо первого уровня. В реальных условиях чаще всего происходит самоуправление или саморегулирование, которое выражается в изменении скорости движения транспортного потока и пропускной способности. Многие рассогласования могут быть устранены службой содержания и ремонта дорог, которая осуществляет управляющие воздействия второго уровня. При возрастании интенсивного движения и воздействий погодно-климатических условий, которые постепенно накапливаются, приводят к ухудшению состояния дороги, управляющие воздействия второго уровня не обеспечивают нормального функционирования. Требуется управляющее воздействие первого уровня - капитальный ремонт или реконструкция дороги, что приводит к изменению постоянных параметров и основных транспортно-эксплуатационных характеристик лесовозных автомобильных дорог, т.е. параметров аппарата обслуживания.

В процессе проектирования дороги определяют оптимальные технические нормативы и транспортно-эксплуатационные показатели, обеспечивающие данный спрос с требуемым качеством обслуживания, т.е. требуемую пропускную способность, скорость и безопасность движения, себестоимость перевозок в эталонных или других, принятых за нормативные, условиях движения. Для обеспечения принятых в нормах выходных характеристик функционирования комплекса $-\hat{x}$ в процессе эксплуатации должны быть определены необходимые мощности и ресурсы дорожнотранспортной службы, методы организации движения, очередность и виды производств дорожнотранспортных работ, т.е. нормативные характеристики процесса обслуживания. При этом, учитывая принцип накопленного воздействия вектор $\hat{x}(t)$ изменяется по закону

$$
\hat{x}(t)=\int_{0}^{t} \Psi[\hat{x}(0), \omega(t), N(t)] d t
$$

То есть на вектор $\hat{x}$ векторы $\omega$ и $N$ оказывают интегральное воздействие, поэтому векторы $\hat{x}$ и $x$ должны учитывать не только текущее значение $\omega$ и $N$, но и их накопленный эффект.

Затем с учетом фактических транспортных потоков и погодно-климатических условий опреде- 
ляют фактические проектные параметры и транспортно-эксплуатационные характеристики дороги, которые должны обеспечить требуемые выходные параметры функционирования комплекса $x$. Для обеспечения проектных параметров и характеристики в процессе эксплуатации определяются проектные мощности и ресурсы дорожно-эксплуатационной службы.

Проектирование обычно идет методом подбора оптимального варианта, путем определения и сравнения рассогласований $x$ - $\hat{x}$, т.е. отличия проектных значений выходных параметров аппарата обслуживания от нормативных.

На основании сравнения и анализа рассогласований производится вычисление оптимального управления I уровня ОУ1, таким образом, переменными управлениями первого уровня являются основные технические параметры дорог и транспортно-эксплуатационные характеристики, а также мощность и ресурсы дорожно-эксплуатационной службы.

На втором уровне осуществляется текущее, оперативное управление функционированием процесса обслуживания в рамках номинальных параметров процесса обслуживания, определенных для большого выбора типичных условий окружающей среды и характеристик транспортного потока. Расчет набора основных управляющих воздействий второго уровня должен быть составной частью проектирования лесовозной автомобильной дороги. В качестве нормативной базы для управления на втором уровне принимаются расчетные состояния переменных параметров дорожных условий, проектные схемы организации движения и содержания лесовозной автомобильной дороги, обеспечиваю-

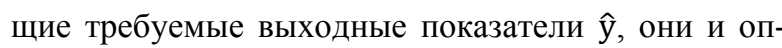
ределяют нормативные характеристики процесса обслуживания.

Существенное влияние на колебания характеристик процесса обслуживания имеют технические параметры аппарата обслуживания $X 1, X 3$, т.е. технический уровень лесовозной автомобильной дороги, заложений в проекте.

Под воздействием лесовозного транспортного потока, метеорологических условий и уровня содержания, формируется фактическое состояние лесовозной автомобильной дороги и, прежде всего, ее переменных параметров, реализуются фактические схемы и методы организации движения - фактические характеристики процесса обслуживания, выходные параметры которого у и есть фактические показатели функционирования комплекса ВАДС.

В сумматоре эти показатели сравниваются с нормативными и определяются рассогласования

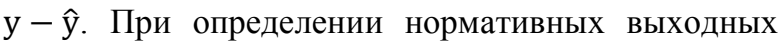
параметров процесса обслуживания учитываются накопленные воздействия, которые зависят от срока службы дороги, ее технических параметров, погодно-климатических условий и уровня содержания.

$$
\hat{\mathrm{y}}(t)=\varphi[\hat{\mathrm{y}}(0), \omega(t), f(t), u(t), x(t)] .
$$

Весьма важное значение на втором уровне управления имеет оперативное прогнозирование ожидаемого изменения условий движения для своевременного принятия соответствующих мер по предупреждению возможных рассогласований.

Предложенная модель управления системой «дорожные условия -транспортные потоки» позволяет обеспечивать функционирование комплекса в любых условиях погоды и климата. Она показывает роль и значение совершенствования подсистемы «дорожные условия», а также системную целостность взаимодействия всего комплекса ВАДС в процессе функционирования. Отсюда вытекает системная целостность и неразрывность процесса создания и функционирования дороги.

Анализ предложенной модели и целевого функционала показывает, что оптимизация функционирования системы ДУ-ТП может идти по многим направлениям, главным из которых являются: оптимальное развитие и размещение сети лесовозных автомобильных дорог, совершенствование их технического уровня и уровня содержания, инженерное оборудование дорог, эффективное управление дорожным движением, исключая рациональное распределение лесовозных транспортных потоков по сети лесовозных автомобильных дорог и управление режимами на отдельных лесовозных автомобильных дорогах и участках лесовозных автомобильных дорог. 


\section{Лесоинженерное дело}

Разработанная модель позволяет осуществить теоретический анализ и экспериментальную проверку взаимодействия систем комплекса ВАДС с целью выявления резервов повышения эффективности его функционирования и целенаправленного управления этим процессом. Транспортноэксплуатационные характеристики лесовозных автомобильных дорог являются главными факторами, которые формируют условия движения лесовозов на дороге, режим, удобство и безопасность движения и определяют эффективность работы автомобильного транспорта, причем связи многих характеристик дорог с режимом движения носят детерминированный, коррекционный характер. Эти закономерности являются базой для разработки технических требований к лесовозным автомобильным дорогам.

Предложенная модель комплексного создания и управления функционированием лесовозной автомобильной дороги может внедриться уже в настоящее время. При проектировании дорог целесообразно предусматривать комплекс управляющих инженерных и организационных мероприятий, обеспечивающих расчетные условия движения в процессе всего периода эксплуатации дороги, независимо от периода года и погодно-климатических условий.

Оценка эффективности принятых решений должна производиться с обязательным учетом обеспечения требуемых показателей работы лесовозной автомобильной дороги в неблагоприятные периоды года и в сложных погодных условиях. Однако далеко не всегда экономически целесообразно обеспечивать техническое совершенство лесовозной автомобильной дороги в расчете на редко повторяющийся спрос на обслуживание, например, маловероятную пиковую интенсивность движения, или на редкое метеорологическое явление: сильный ливень или шторм. Кроме того, невозможно в короткие сроки усовершенствовать сеть существующих лесовозных автомобильных дорог, поэтому необходима разработка широкого спектра рациональных методов управления движения лесовозных транспортных потоков с целью повышения эффективности работы лесовозного автомобильного транспорта, удобства и безопасности движения в реальных дорожных и природно-климатических условиях [2].

Предложенная модель управления функционированием систем ДУ-ТП позволяет решить эту задачу в каждом конкретном случае с использованием методов системного анализа и теории исследования операций [8].

Успех управления системой ДУ-ТП зависит от трех групп факторов. К заданным заранее известным факторам и условиям можно отнести характеристики транспортного потока $£_{1}, £_{2}, £_{3} \ldots £_{n}$. К зависящим от нас факторам или элементам решения, которые могут пригодиться, относятся геометрические параметры, транспортноэксплуатационные характеристики, показатели уровня ремонта и содержания, организации и управления движением - $\beta_{1}, \beta_{2} \ldots \beta_{n}, \quad \gamma 1, \quad \gamma 2$ .. $\gamma n$.Третья группа - это неизвестные факторы: метеорологические условия $X_{1}, X_{2} \ldots \ldots, X_{n}$, значения которых заранее не предсказуемы.

Как указывает Вентцель Е.С., такая задача относится к классу задач о принятии решения в условиях неопределенности [8]. Учитывая это, предложена ниже следующая математическая формулировка задач управления системой ДУ-ТП.

При заданных характеристиках транспортного потока $\alpha_{1}, \alpha_{2}, \ldots . a_{n}$ в пределах изменения теоретических параметров, транспортноэксплуатационных характеристик лесовозных автомобильных дорог, организации работ по ремонту, содержанию и организации движения в рассматриваемых вариантах.

$$
\begin{aligned}
& \beta_{1}^{\min }, \ldots, \beta_{1}^{\max }, \ldots, \beta_{\mathrm{n}}^{\min }, \ldots, \beta_{\mathrm{n}}^{\max }, \ldots, \gamma_{1}^{\min }, \ldots, \gamma_{1}^{\max }, \\
& \ldots, \gamma_{\mathrm{n}}^{\min }, \ldots, \gamma_{\mathrm{n}}^{\max }
\end{aligned}
$$

С учетом основных характеристик метеорологических условий $X_{1}, X_{2}, \ldots, X_{n}$, заданных распределениями вероятностей появления

$$
\begin{aligned}
& P_{1}=f\left(x_{1}\right), P_{2}=f\left(x_{2}\right), \ldots, P_{n}=f\left(x_{n}\right) \\
& \left(P=\left[P_{i j}\right] x \ldots x_{n}\right)
\end{aligned}
$$

и зависимости изменения состояния дорог, режима и безопасности движения от интенсивности метеорологических явлений,

$\beta_{t}=f_{1}\left(x_{1}, x_{2}, \ldots, x_{n}\right) ; \gamma_{t}=f_{2}\left(x_{1}, x_{2}, \ldots, x_{n}\right)$, 
a также с учетом ограничений по затратам на строительство, ремонт и содержание дорог

$$
\varphi_{r 1}\left(\gamma_{1}, \gamma_{2}, \ldots, \gamma_{n}\right) \leq C_{r 1},\left(r_{1}=1,2, \ldots, m\right) ;
$$

$\varphi_{r 2}\left(\beta_{1}, \beta, \ldots, \beta\right) \leq C_{r 2},\left(r_{2}=1,2, \ldots, m\right)$;

и дополнительных ограничений по некоторым выходным параметрам функционирования (II, $\mathrm{V}, \mathrm{P}, \mathrm{K}_{\mathrm{a}}$ ) определить такие значения параметров $\beta_{l}$, $\beta_{2}, \ldots, \beta_{n} u \gamma_{1}, \gamma_{2}, \ldots, \gamma_{n}$, которые по возможности обеспечивают экстремальные значения целевой функции

$$
\begin{aligned}
& E=f\left(£_{1}, £_{2}, £_{3} \ldots £_{n}, \beta, \beta_{2}, \beta_{3} \ldots \beta_{n}, \gamma_{1}, \gamma_{2}, \gamma_{3} \ldots \gamma_{n},\right. \\
& \left.x_{1}, x_{2}, x_{3} \ldots x_{n}\right)
\end{aligned}
$$

Критерий эффективности для указанной задачи $E=\min$.

Поскольку известны только законы распределения параметров метеорологических условий и вероятности их появления, а не конкретные данные о величинах этих параметров, не может быть единственного оптимального решения поставленной задачи, но может быть определена область приемлемых решений, в пределах которой может быть выбрано окончательно с учетом различных ограничений. Такова в общем виде математическая модель управления функционирования системы ДУТП.

Необходимо разработать и ряд дополнительных ограничений и требований, учитывающих роль автомобильно-дорожной системы не только в экономической и хозяйственной деятельности, но и в культурно-бытовой и социальной жизни общества.
С этой точки зрения представляется целесообразным разделить все лесовозные автомобильные дороги по степени обеспеченности движения в период неблагоприятных погодных условий (исключая стихийные бедствия) на две группы:

1 группа - лесовозные автомобильные дороги, по которым перерывы движения не допускаются в течение всего срока эксплуатации, а снижение скоростей движения и пропускной способности в неблагоприятных погодных условиях не должно превышать нормативных требований;

2 группа - лесовозные автомобильные дороги, по которым допускаются перерывы (закрытие) движения не более установленной нормативной длительности при метеорологических явлениях выше определенной интенсивности. Такой подход позволяет уточнить требования к выбору критерия оценки эффективности функционирования дорог и дополнить их требованиями, которые во многих случаях могут иметь решающее значение при выборе инженерных, организационных и других управляющих решений. С учетом выполненного анализа представляется целесообразным предложить критерии оптимальности функционирования лесовозной автомобильной дороги и дополнитель-

\begin{tabular}{|c|c|c|}
\hline Показатели & Дороги I группы & Дороги II группы \\
\hline $\begin{array}{l}\text { Суммарные } \quad \text { приведенные } \text { дорожно- } \\
\text { транспортные затраты E }\end{array}$ & $\mathrm{E}=\min$ & $\mathrm{E}=\min$ \\
\hline Пропускная способностьII & $\mathrm{I} \geq \mathrm{N}, \mathrm{V} \geq \mathrm{V}_{\mathrm{M}}$ & $\mathrm{II} \geq \mathrm{N}, \mathrm{II}=0$ \\
\hline $\begin{array}{l}\text { Скорость движения в неблагоприятных } \\
\text { метеорологических условиях }\end{array}$ & $\begin{array}{c}\left(\mathrm{V}_{\mathrm{M}}-\text { допускаемый уровень снижения }\right. \\
\text { скорости })\end{array}$ & $\mathrm{V}=0$ \\
\hline Длительность перерыва движения Р & $\mathrm{P}=0$ & $\begin{array}{c}\mathrm{P}<\mathrm{P}_{\mathrm{m}}\left(\mathrm{P}_{\mathrm{m}}-\text { допустимая длительность }\right. \\
\text { перерыва движения })\end{array}$ \\
\hline $\begin{array}{l}\text { Максимальный суммарный коэффици- } \\
\text { ент аварийности по сезонам года К }\end{array}$ & $\mathrm{K}_{\mathrm{a}} \leq \mathrm{K}_{\mathrm{am}}$ & $\mathrm{K}_{\mathrm{a}} \leq \mathrm{K}_{\mathrm{am}}$ \\
\hline
\end{tabular}
ные требования и допущения, указанные в табл. 1.

Критерии оптимальности функционирования лесовозных автомобильных дорог 


\section{Лесоинженерное дело}

Таким образом, необходимым и достаточным условием функционирования лесовозной автомобильной дороги является обеспечение минимума приведенных дорожно-транспортных затрат на обеспечение движения транспортных потоков с установленными скоростями, непрерывностью, пропускной способностью и безопасностью движения в неблагоприятные периоды года и в сложных погодных условиях.

Разработанная модель управления системой «дорожные условия - транспортные потоки» позволяет осуществить теоретический анализ и экспериментальную проверку взаимодействия систем комплекса «водитель - автомобиль - дорога среда» с целью выявления резервов повышения эффективности его функционирования и целенаправленного управления этим процессом. Она может быть внедрена уже в настоящее время. Однако при проектировании дорог необходимо также предусматривать комплекс управляющих инженерных и организационных мероприятий, обеспечивающих расчетные условия движения в процессе всего периода эксплуатации дороги, независимо от периода года и погодно-климатических условий.

\section{Библиографический список}

1. Иванников, В. А. Моделирование информационных систем грузопотоков на лесозаготовительных предприятиях [Текст] / В. А. Иванников, А. В. Быков, А. С. Сушков // Строительные и дорожные машины. 2012. - № 2. - C. 24-28.

2. Козлов, В. Г. Методы, модели и алгоритмы проектирования лесовозных автомобильных дорог с учетом влияния климата и погоды на условия движения [Текст] : дис. ... д-ра техн. наук / В. Г. Козлов. Архангельск : САФУ, 2017. - 406 с.

3. Автоматизированный расчет загрязнения атмосферы токсичными компонентами отработанных газов [Текст] / В. К. Курьянов [и др.] // Депонированная рукопись № 561-В2003 28.03.2003.

4. Повышение эффективности функционирования системы «Водитель-Автомобиль-Дорога-Среда» в лесном комплексе [Текст] / В. К. Курьянов [и др.]. - М.: «Флинта» : «Наука», 2010. - 130 с.

5. Моделирование грузовых потоков на магистралях с учетом меняющихся дорожных условий для агропромышленного комплекса [Текст] / В. Г. Козлов [и др.] // Системный анализ и моделирование процессов управления качеством в инновационном развитии АПК : материалы международной научно-практической конференции, Воронеж: ФГБОУ ВПО «ВГУИТ», 8-9 апреля 2015 г. - С. 612-624.

6. Повышение эффективности функционирования автомобильных дорог лесного комплекса [Текст] : моногр. / В. Г. Козлов [и др.]. - Йошкар-Ола, 2016.

7. Вентцель, Е. С. Теория вероятностей [Текст] : учеб. / Е. С. Вентцель. - 11-е изд., стер. - М. : КНОРУС, 2010. $-664 \mathrm{c}$.

8. Berestnev O., Soliterman Y., Goman A. International Symposium on History of Machines and Mechanisms Proceedings 2000. P. 325-332.

9. Park K. [et al.] Journal of Construction Engineering and Management 2003129 (1) 25-31.

10. Skrypnikov A. V., Dorokhin S. V., Kozlov V. G., Chernyshova E. V. Mathematical model of statistical identification of car transport informational provision // Journal of Engineering and Applied Sciences. 2017. No. 2. P. 511-515.

\section{References}

1. Ivannikov V.A., Bykov A.V., Sushkov A.S. Modelirovaniye informatsionnykh sistem gruzopotokov na lesozagotovitelnykh predpriyatiyakh // Stroitelnyye i dorozhnyye mashiny. - 2012. - № 2. - S. 24-28.

2. Kozlov V.G. Metody, modeli $i$ algoritmy proyektirovaniya lesovoznykh avtomobilnykh dorog s uchetom vliyaniya klimata i pogody na usloviya dvizheniya: disser. ... dokt. tekhn. nauk. Arkhangelsk: SAFU. 2017. 406 s. 
3. Kuryanov V. K. The Automated calculation of air pollution by toxic components of exhaust gases. / Kuryanov V. K. etc. // Deposited manuscript No. 561-2003 28.03.2003.

4. Kurianov. V.K. Povysheniye effektivnosti funktsionirovaniya sistemy «Voditel-Avtomobil-Doroga-Sreda» v lesnom komplekse / V.K. Kurianov. O.V. Ryabova. E.V. Kondrashova. A.V. Skrypnikov. A.Yu. Chuvenkov. Moskva: izd-vo «Flinta». «Nauka». 2010. $130 \mathrm{~s}$.

5. Modelirovaniye gruzovykh potokov na magistralyakhs uchetom menyayushchikhsya dorozhnykh usloviy dlya agropromyshlennogo kompleksa / V.G. Kozlov. Chan Van Zy. M.M. Umarov. A.Yu. Arutyunyan. E.V. Chernyshova // Sistemnyy analiz i modelirovaniye protsessov upravleniya kachestvom v innovatsionnom razvitii APK : materialy mezhdunarodnoy nauchno-prakticheskoy konferentsii. Voronezh: FGBOU VPO «VGUIT». 8-9 aprelya 2015 g. S. 612-624.

6. Povysheniye effektivnosti funktsionirovaniya avtomobilnykh dorog lesnogo kompleksa: monografiya / Kozlov V.G.. Smirnov M.Yu.. Skrypnikov A.V.. Dorokhin S.V.. Skvortsova T.V.. Zhuravlev I.N. Yoshkar-Ola. 2016.

7. Teoriyaveroyatnostey : uchebnik / E.S. Venttsel. 11 ed,. ster. M. : KNORUS. 2010. 664 s.

8. Berestnev O. Soliterman Y. and Goman A 2000 International Symposium on History of Machines and Mechanisms Proceedings. 325-332.

9. Park K. Hwang Y. Seo S. Asce M and Seo H 2003 Journal of Construction Engineering and Management 129 (1). 25-31.

10. Skrypnikov A V. Dorokhin S V. Kozlov V G and Chernyshova E V 2017 Mathematical model of statistical identification of car transport informational provision Journal of Engineering and Applied Sciences 2. 511-515.

\section{Сведения об авторах}

Козлов Вячеслав Геннадиевич - доцент кафедры эксплуатации транспортных и технологических машин ФГБОУ ВО «Воронежский государственный аграрный университет имени императора Петра I», кандидат технических наук, доцент, г. Воронеж, Российская Федерация; e-mail: vya-kozlov@ yandex.ru.

Скрыпников Алексей Васильевич - профессор кафедры информационной безопасности ФГБОУ ВО «Воронежский государственный университет инженерных технологий», доктор технических наук, профессор, г. Воронеж, Российская Федерация; e-mail: skrypnikovvsafe@ mail.ru

Микова Елена Юрьевна - экстерн кафедры информационной безопасности ФГБОУ ВО «Воронежский государственный университет инженерных технологий», г. Воронеж, Российская Федерация, е-таil: Leencha@ya.ru

Могутнов Роман Викторович - экстерн кафедры информационной безопасности ФГБОУ ВО «Воронежский государственный университет инженерных технологий», г. Воронеж, Российская Федерация, e-mail: r-mogutnov@mail.ru

Чирков Евгений Викторович - экстерн кафедры информационной безопасности ФГБОУ ВО «Воронежский государственный университет инженерных технологий», г. Воронеж, Российская Федерация, e-mail: chirkov_e_v@mail.ru

\section{Information about authors}

Kozlov Vyacheslav Gennadievich - Associate Professor of Department of exploitation of transport and technological machines, Federal State Budget Education Institution of Higher Education " Voronezh State Agrarian University named after Emperor Peter the Great", Ph.D. in Engineering, Associate Professor, Voronezh, Russian Federation; e-mail: vya-kozlov@yandex.ru. 


\title{
Лесоинженерное дело
}

Skrypnikov Alexey Vasilyevich - Professor, Department of information security of the "Voronezh state University of engineering technologies", doctor of technical Sciences, Professor, Voronezh, Russian Federation; e-mail: skrypnikovvsafe@mail.ru

Mikova Elena Y. - external information security department FSBEI HE"Voronezh State University of Engineering Technologies", Voronezh, Russian Federation,e-mail: Leencha@ya.ru

Mogutnov Roman Viktorovich - external information security department FSBEI HE "Voronezh State University of Engineering Technologies", Voronezh, Russian Federation,e-mail: r-mogutnov@ mail.ru

Chirkov Evgeniy Viktorovich - external information security department FSBEI HE "Voronezh State University of Engineering Technologies" , Voronezh, Russian Federation,e-mail:chirkov_e_v@mail.ru

DOI: 10.12737/article_5ab0dfc0247508.69266095

УДК $630 * 7$

\section{ОБОСНОВАНИЕ ТЕХНОЛОГИИ ЗАГОТОВКИ ДРЕВЕСИНЫ С ПОМОЩЬЮ ИМИТАЦИОННОГО МОДЕЛИРОВАНИЯ НА СЕТЯХ ПЕТРИ}

\author{
доктор технических наук, доцент А. П. Соколов ${ }^{1}$
}

Е. В. Осипов ${ }^{1}$

1 - ФГБОУ ВО «Петрозаводский государственный университет», г. Петрозаводск, Российская Федерация

Статья посвящена описанию решения задачи обоснования технологиизаготовки древесины, с использованием имитационного моделирования производственных процессов на сетях Петри.Рассматривается возможность использования специально разработанных имитационных моделей работы комплексов лесозаготовительных машин, состоящих из харвестера и форвардера, не только для принятия решений по выбору машин при формировании комплексов, но и для обоснования технологических решений. Решение задачи описывается на примере делянки неправильной формы с магистральным волоком значительной протяженности. Основу применяемого подхода составляет использование метода дискретно-событийного имитационного моделирования на сетях Петри. Приведены результаты выполненных полевых исследований, моделирования и совершенствования технологического процесса. Результаты моделирования показали, что в условиях рассмотренной лесосеки форвардер загружен неравномерно. Простой этой машины составил 24,5 ч. или $7 \%$ времени. Простой харвестера составил 9 ч. или $3 \%$.В результате анализа полученного при моделировании распределения простоевмашин во времени, было выявлено, что это связано с постоянно уменьшающейся производительностью форвардера, вследствие увеличивающегося расстояния трелевки. В результате выполненного исследования было рекомендовано внести изменениев технологию разработки лесосеки, заключающееся в том, что харвестер будет начинать работу не с ближних по отношению к погрузочной площадке пасек, а с дальних, находящихся в конце магистрального волока.Моделирование, выполненное после внесения в модель изменений, показало, что в случае перехода к предлагаемой технологии разработки лесосек, может быть достигнут рост производительности комплекса машин харвестер+форвардер на 2,3\% за счет сокращения суммарного простоя машин. Можно рекомендовать применение такой технологии в случае лесосек с магистральным волоком значительной протяженности. Таким образом, при решении реальной производственной задачи была доказана эффективность предлагаемого подхода к оценке вариантов технологических решений на заготовке древесины.

Ключевые слова: заготовка древесины, системы лесозаготовительных машин, харвестер, форвардер, имитационное моделирование, сети Петри 